\title{
Erratum to: Psychometric Properties of the WHOQOL-BREF in an Iranian Adult Sample
}

\author{
A. R. Yousefy • Gh. R. Ghassemi $\cdot$ N. Sarrafzadegan • \\ S. Mallik $\cdot$ A. M. Baghaei $\cdot$ K. Rabiei
}

Published online: 21 February 2010

(C) Springer Science+Business Media, LLC 2010

\section{Erratum to: Community Ment Health J \\ DOI 10.1007/s10597-009-9282-8}

One of the co-author's name had been wrongly published. The correct version is A. R. Yousefy and not A. R. Usefy as published.

The online version of the original article can be found under doi:10.1007/s10597-009-9282-8.

\footnotetext{
A. R. Yousefy

Medical Education Research Centre (MERC), Isfahan University

of Medical Sciences, Isfahan, Iran

Gh. R. Ghassemi ( $₫)$

Department of Psychiatry, School of Medicine, Isfahan

University of Medical Sciences, Isfahan, Iran

e-mail: ghassemi@med.mui.ac.ir; zcswei@gmail.com

\section{N. Sarrafzadegan}

Isfahan Cardiovascular Research Centre, Isfahan University

of Medical Sciences, Isfahan, Iran

\section{S. Mallik}

Zenderood Clinic of Social Work, Isfahan, Iran

\author{
A. M. Baghaei \\ Isfahan Cardiovascular Research Centre, WHO Collaborating \\ Centre, Isfahan University of Medical Sciences, Isfahan, Iran \\ K. Rabiei \\ Cardiac Rehabilitation Department, Isfahan Cardiovascular \\ Research Centre, WHO Collaborating Centre, Isfahan University
}

of Medical Sciences, Isfahan, Iran
} 